oedema is best seen when a cardiac MR scan is carried out within 14 days of the initial Troponin positive event and normally subsides after 6 weeks. Access to CMR during the COVID pandemic year was restricted at the height of the pandemic.

Purpose The purpose of this study was three fold. 1. To examine what proportion of patients with a rise and fall in Troponin and unobstructed coronary arteries were offered a CMR scan in our hospital during the year of 2020. 2. To examine the waiting times for a CMR scan during this year 3. To establish in what proportion of patients the CMR scan changed the diagnosis and management of the patients.

Methods This is a retrospective cohort study of all patients that presented to the Royal Berkshire Hospital with chest pain, a troponin rise and unobstructed coronaries on invasive angiogram between January and December of 2020. We collected data on demographics, the degree of troponin rise, echocardiography results, the discharge diagnosis and details of CMR findings (if performed). For quantitative data analysis, the number of days between presentation and CMR imaging was collated, as was the incidence of change of diagnosis and or management.

Results Complete data was available for 104 patients who presented to the Royal Berkshire Hospital with chest pain between 6th January and 21st December 2020. All underwent coronary angiography. Of the patients included, 55.8\% (58) were men and $44.2 \%$ (46) were women - all between 29 and 89 years of age. $61 \%$ of these patients had an ECHO as an inpatient, whilst a further $26 \%$ had a Vscan performed. Overall $87 \%$ of these patients had a Vscan or ECHO. Of the patients meeting the criteria, 31\% of them received an inpatient (16\%) or urgent outpatient (84\%) CMR, with the average time between presentation and image being 47.3 days. Finally, 59\% of diagnosis made on discharge were altered by findings on CMR, with $47 \%$ of management plans changed as a result of findings. Final diagnoses, following CMR, can be seen in figure 1. Subgroup analyses were performed on those that displayed initial raised troponins that remained static during admission, and those that received discharge diagnoses of Takotsubo or myocarditis/ pericarditis/ myopericarditis.

Conclusions During 2020, the majority of patients (70\%) with Troponin rise and unobstructed coronary arteries did not undergo CMR scanning in our hospital. Of the patients offered a CMR scan during the year of 2020, the scan

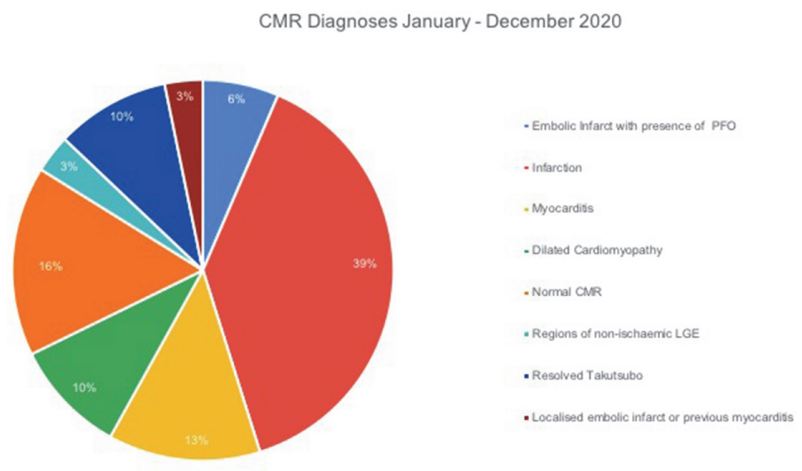

Abstract 174 Figure 1 changed the diagnosis in the majority of patients $(59 \%)$ but the wait for CMR was considerable (average of 47.3 days). This study suggests that in 2020, in our hospital the majority of patients may have ended up with suboptimal treatment following an admission with chest pain, troponin rise and unobstructed coronary arteries due to reduced access to CMR. This may be, in part, down to the impact of COVID on access to CMRs during the height of the pandemic.

Conflict of Interest Nil

\section{CT FRACTIONAL FLOW RESERVE: EXPERIENCES FROM A DISTRICT GENERAL HOSPITAL}

Hazhee Rasoul, Saad Fyyaz, Daniela Noakes, Carl Shakespeare, Sarojini David, Zeeshan Khawaja, Nikolaos Papamichael, Khaled Al-Fakih. Lewisham and Greenwich NHS Trust, London, UK

\subsection{6/heartjnl-2021-BCS.172}

Introduction The UK National Institute for Health and Care Excellence recommended CT fractional flow reserve (CTFFR) to assess the functional significance of stenoses found on CT coronary angiogram (CTCA). NHS England subsequently negotiated a UK wide contract with HeartFlow, the provider of CTFFR, in 2018. We describe our experience in a district general hospital.

Method All patients referred for CTFFR following CTCA between 1st January 2019 and 31st March 2020 were analysed. CTCA stenosis severity was categorised as mild (30$50 \%)$, moderate $(50-70 \%)$ and severe $(>70 \%$ or left main stem $>50 \%)$. CTFFR $<0.8$ was deemed positive. Cost analysis was undertaken with the following tariffs: CTCA $£ 220$, CTFFR $£ 530$, invasive coronary angiogram (ICA) $£ 1000$, invasive FFR $£ 336$.

Results 140 patients were referred for CTFFR, with 15 (11\%) rejected due to image quality (figure 1). 125 were analysed (80 male, mean age 59 yrs). 44 patients had mild stenosis on CTCA, 43 of which had negative CTFFR. 1 patient had positive CTFFR but did not attend follow up. 81 patients had moderate/severe stenosis on CTCA: 36 had positive CTFFR: 6 were medically managed, 29 underwent ICA, with 22 (76\%) revascularised. 4 patients had false positive CTFFR and were medically managed after ICA. 6 had false negative CTFFR in one vessel, 5 of which had true positive and one had false positive in a different vessel.45 had negative CTFFR: 18 were medically managed, 14 underwent ICA. 4 (29\%) were revascularised, with 3 noted to have calcium scores ranging 6221108. Cost per patient in our cohort was $£ 1166.51$. If only patients with CTCA stenosis $>50 \%$ underwent CTFFR the cost was $£ 971.95$.

Conclusion CTFFR can be an effective gatekeeper to ICA with revascularisation rates of $76 \%$ in CTFFR $<0.8$ versus $29 \%$ with CTFFR $>0.8$. However, a high calcium score may influence CTFFR accuracy and further investigation is warranted in these patients. The use of CTFFR with mild CTCA stenosis did not influence management, with significant cost savings of $£ 194.56$ per patient had they not been referred for CTFFR.

Conflict of Interest None declared 


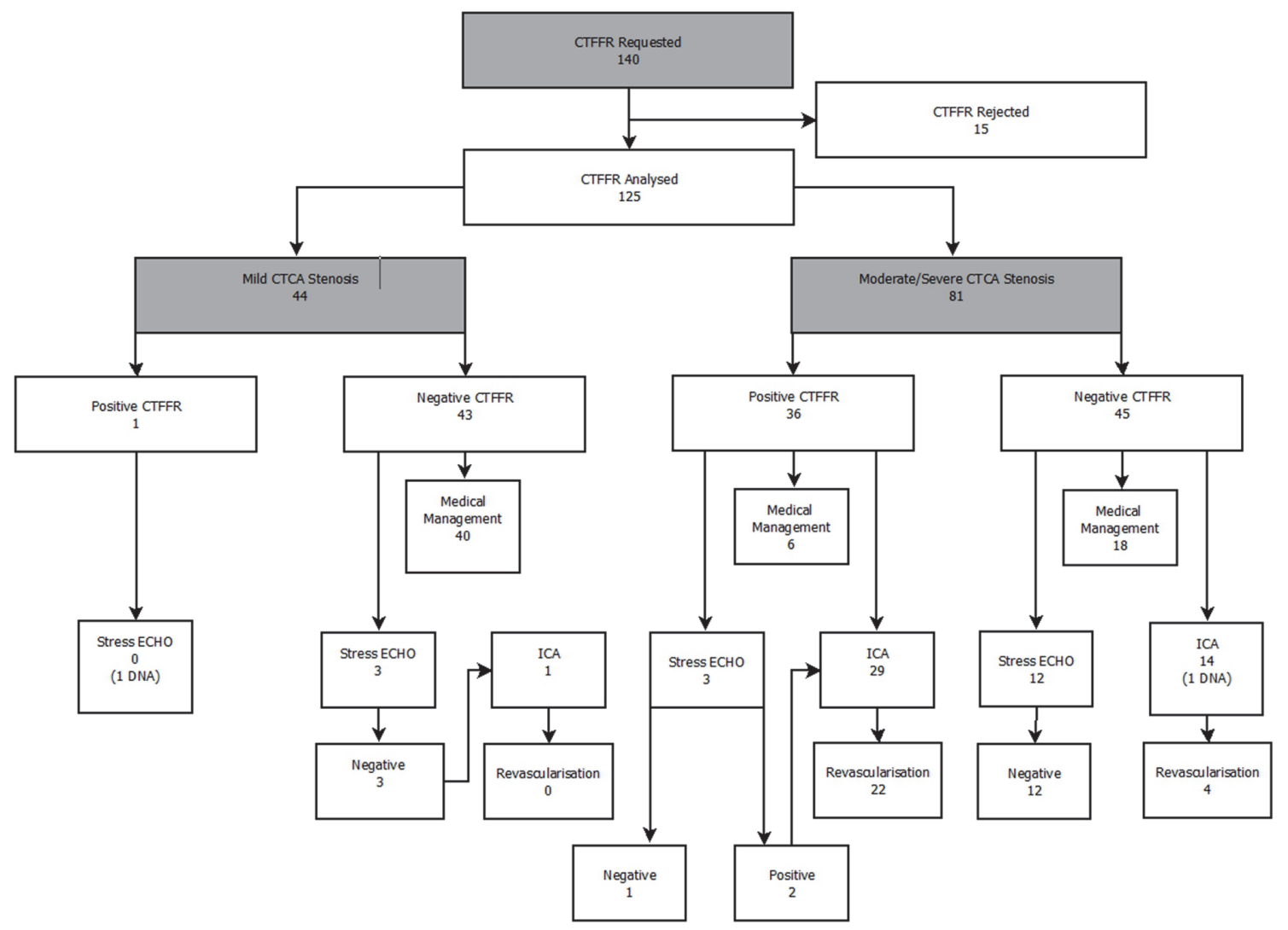

Abstract 175 Figure 1 Downstream investigations and outcomes of patients following CTFFR analysis. CTFFR = CT Fractional Flow Reserve; CTCA = CT Coronary Angiogram; ICA = Invasive Coronary Angiogram

\section{COVID-19 DISEASE AND CARDIAC INVOLVEMENT - A LOCAL EXPERIENCE}

${ }^{1}$ Muhammad Raheemuddin Ahmed, ${ }^{2}$ Samsul Islam, ${ }^{2}$ Emily Challinor, ${ }^{2}$ Tom Ingram, ${ }^{2}$ Arif Khan. ${ }^{1}$ Royal Shrewsbury hospital, Shrewsbury, UK; ${ }^{2}$ Shrewsbury and Telford NHS Trust

\subsection{6/heartjnl-2021-BCS.173}

Aims The aim of this review to assess cardiac involvement in patients with severe COVID-19 patients. We review all patients with COVID 19 disease admitted in our trust requiring transthoracic echocardiograms on their clinical indications.

Background Cardiac involvement in COVID-19 disease has been found to be prognostic factor and has been related with higher mortality and morbidity. In a large series with COVID-19 those with heart disease had a fatality rate around $10.5 \% .^{12}$

Methods All adult patients who were COVID-19 positive on PCR admitted between March 2020 and February 2021, who had an echocardiogram, were identified through our local database. Their demographics, co-morbid, troponin levels and Pro NT-BNP were analysed. All echocardiograms reports which were finalised by the imaging cardiologist were included in our analysis.

Results There were a total of 41 patients who had echocardiograms during their stay in the hospital with COVID-19 disease. Mean age was 70 (range 45-90) years old. There were $70 \%$ male and $30 \%$ female patients. $12 \%$ were diabetic, $49 \%$ hypertensive and $40 \%$ had previous heart disease. Pulmonary embolism diagnosed in $10 \%$ of patients by CT pulmonary angiogram. $56 \%$ of patients required high flow oxygen and
$21 \%$ need mechanical ventilation. Almost all patients had troponin and CRP levels on admission. Mean troponin level 215 and mean CRP levels were 197. Mean D dimer levels 1130, and mean creatinine levels were 138. 92\% had evidence of lung involvement in chest X-ray. $13 \%$ patients had new evidence of a diagnosis of left ventricular dysfunction on echocardiography. Similarly, 27\% had a new diagnosis of right ventricular dysfunction. Mean left ventricular diastolic dimension were $4.6 \mathrm{~cm}$ and systolic dimension. $2 \%$ had echo diagnosis of left ventricular thrombus echocardiographic studies. Mean PA pressure on echocardiography were $35 \mathrm{mmHg}$ and mean E/A ratio was 1.2. $17 \%$ of patients were found to have pericardial effusion but none causing haemodynamic compromise.

Conclusion This data suggests high incidence of right and left ventricular involvement in patients with severe COVID-19 disease. We recommend that all patients with COVID-19 disease admitted to hospital and requiring oxygen should have transthoracic echocardiograms during their admission.

Conflict of Interest none

\section{REFERENCES}

1. Zaim S, Chong JH, Sankaranarayanan V, Harky A. COVID-19 and Multiorgan Response. Curr Probl Cardiol 2020;45(8):100618. doi:10.1016/j. cpcardiol.2020.100618

2. Tomasz J Guzik, Saidi A Mohiddin, Anthony Dimarco, Vimal Patel, Kostas Savvatis, Federica M Marelli-Berg, Meena S Madhur, Maciej Tomaszewski, Pasquale Maffia, Fulvio D'Acquisto, Stuart A Nicklin, Ali J Marian, Ryszard Nosalski, Eleanor C Murray, Bartlomiej Guzik, et al. COVID-19 and the cardiovascular system: implications for risk assessment, diagnosis, and treatment options. Cardiovascular Research, 1 August 2020;116(10):1666-1687 